\title{
A ESPIRITUALIDADE E O CUIDAR EM ENFERMAGEM EM TEMPOS DE PANDEMIA
}

Diogo Jacintho Barbosa ${ }^{1}$ Marcia Pereira Gomes ${ }^{2}$ Antônio Marcos Gomes Tosolit ${ }^{1}$ Fabiana Barbosa Assumpção de Souza ${ }^{2}$

\author{
https://orcid.org/0000-0001-8816-1770 \\ https://orcid.org/ 0000-0002-7872-5891 \\ https://orcid.org/0000-0003-4235-9647 \\ http//orcid.org/0000-0001-8098-5417
}

Objetivo: Refletir sobre a espiritualidade como suporte para o profissional de enfermagem em tempos de pandemia durante o cuidado prestado à clientela. Método: Trata-se de um estudo qualitativo descritivo, tipo análise reflexiva. Resultados: A espiritualidade tem se revelado um dos principais recursos dos profissionais e da sociedade para compreender os sofrimentos e fortalecer a humanidade para novos desafios como a pandemia atual. A valorização da dimensão espiritual do paciente pode colaborar com a sua recuperação, melhor entendimento e aceitação da sua condição atual, contribuindo para um melhor equilibrio e qualidade de vida, preservando sua dignidade durante o período de internação. Considerações Finais: Em virtude do papel que a espiritualidade pode ter na doença, vida e qualidade de vida do paciente, é importante que profissionais de enfermagem estejam habilitados a explorar esta faceta dos seus clientes como também em si mesmo. Descritores: Espiritualidade; Infecção por Coronavírus; Cuidado de Enfermagem; COVID-19; Religiosidade.

\section{SPIRITUALITY AND NURSING CARE IN TIMES OF PANDEMIC}

Objective: To reflect on spirituality as a support for nursing professionals in times of pandemic during the care provided to clients. Method: This is a qualitative descriptive study, reflective analysis. Results: Spirituality has proved to be one of the main resources of professionals and society to understand suffering and strengthen humanity for new challenges such as actual pandemic. The appreciation of the patient's spiritual dimension can contribute to his recovery, better understanding and acceptance of his current condition, contributing to a better balance and quality of life, preserving his dignity during the hospitalization period. . Final Considerations: Due to the role that spirituality can play in the patient's disease, life and quality of life, it is important that nursing professionals are able to explore this facet of their clients as well as in themselves. Descriptors: Spirituality; Coronavirus Infection; Nursing Care; COVID-19; Religiosity.

\section{ESPIRITUALIDAD Y CUIDADOS DE ENFERMERÍA EN TIEMPOS DE PANDEMIA}

Objetivo: reflexionar sobre la espiritualidad como un apoyo para los profesionales de enfermería en tiempos de pandemia durante la atención brindada a los clientes. Método: estudio descriptivo cualitativo, análisis reflexivo. Resultados: La espiritualidad ha demostrado ser uno de los principales recursos de los profesionales y la sociedad para comprender el sufrimiento y fortalecer a la humanidad ante nuevos desafíos como la pandemia real. La apreciación de la dimensión espiritual del paciente puede contribuir a su recuperación, una mejor comprensión y aceptación de su condición actual, contribuyendo a un mejor equilibrio y calidad de vida, preservando su dignidad durante el período de hospitalización. Consideraciones de Finales: Debido al papel que puede desempeñar la espiritualidad en la enfermedad, la vida y la calidad de vida del paciente, es importante que los profesionales de enfermería puedan explorar esta faceta de sus clientes, así como en sí mismos. Descriptores: Espiritualidad; Infección por coronavirus; Atención de enfermería, COVID-19; Religiosidad.

Faculdade de Enfermagem da Universidade do Estado do Rio de Janeiro - UERJ, RJ.

2 Universidade Federal do Rio de Janeiro- UNIRIO, RJ.

Autor Correspondente: Diogo Jacintho Barbosa. E-mail: jacinthobarbosa@gmail.com Recebido: 11/5/2020 - Aceito: 20/6/2020 


\section{INTRODUÇÃO}

A doença pelo novo coronavírus (COVID-19) é uma síndrome respiratória aguda grave, causada por um tipo de coronavirus, o 2019-nCoV (Sars-CoV-2) ${ }^{(1)}$, considerada a maior pandemia do século XXI. Até o dia 23 de maio de 2020, foram confirmados 5.175.925 casos da COVID-19, com 338.089 óbitos no mundo. No Brasil foram confirmados 347.398 casos da COVID-19 sendo que 22.013 foram a óbito. Segundo dados internacionais, o Brasil é o quarto em número de casos confirmados e o sexto em número de óbitos ${ }^{(2)}$.

A COVID-19 é uma doença que nos confrontacom a morte e com as questões que são inerentes àesta. A maioria das mortes são inesperadas e agudas e os profissionais que estão na linha de frente devem ser cuidados para evitar colapso físico e mental, uma vez que sua afetação pode interferir no desfecho clínico dos pacientes. Nesse contexto, os suportes físico, psiquico e espiritual devem ser levados em consideração ${ }^{(3)}$. Com relação ao suporte espiritual, percebe-se atualmente a grande influência que a religiosidade e a espiritualidade apresentam sobre a vida dos indivíduos. As questões espirituais e religiosas são capazes de influenciar na forma com que estes se relacionam com os outros, na sua forma de pensar e até mesmo na maneira de lidar com questões do cotidiano(3), como por exemplo, a Pandemia da COVID-19 que assola não só o Brasil, mas o mundo, e expõe os profissionais da saúde ao vírus e ao adoecimento, além de vivenciar muitos óbitos durante seu labor.

Igualmente ao número de casos da COVID-19, tem aumentado em grande escala o reconhecimento de ferramentas que auxiliem no enfrentamento das situações estressantes que podem ser trazidas pela doença. Quando a saúde é gravemente afetada, as pessoas comumente recorrem à religiosidade/espiritualidade, em busca de conforto e recursos para lidar com a situação(4).

Enquanto que a religiosidade está relacionada à ideia de comunidade, a alguma atividade ritualística e à doutrina religiosa, a espiritualidade se relaciona a uma força interior do indivíduo, à construção de sentido para as diversas situações da vida ${ }^{5}$. É intrínseca ao ser humano, e não necessariamente, depende das experiências religiosas e da religiosidade(6).

Entende-se que os profissionais da saúde lidam diariamente não somente com o medo da morte, mas também com as incertezas relacionadas ao tratamento eficaz, às formas de diagnóstico seguro, uso adequado correto e racional dos Equipamentos de Proteção Individual (EPI), influenciando, assim,na saúde mental destes, sobretudo dos profissionais de enfermagem que vem sendo elencados como aqueles que estão na linha de frente no combate à COVID-19. Tais condições de trabalho a que estão expostos os profissionais da saúde, em especial os de Enfermagem, tem afetado além de sua saú- de mental a sua espiritualidade, levando-os a desmotivação de modo a influenciar de maneira direta no cuidado de si e do outro que necessita.

Analisando-se o cuidado prestado por profissionais de enfermagem, foi observado que estes por diversas vezes apresentam sentimentos espirituais que podem ser capazes de potencializar um cuidado humanizado e integral. O fortalecimento e a identificação desses sentimentos auxiliam a equipe a lidar com o sofrimento psicológico oriundo do ambiente de trabalho, sobretudo aqueles mais estressores ${ }^{(7)}$.

Evidenciando as questões relacionadas à atualidade do tema e levando-se em conta as questões relacionadas à espiritualidade para a enfermagem, na perspectiva de explorar esse assunto diante da pandemia atual, este estudo teve como objetivo refletir sobre a espiritualidade como suporte para o profissional de enfermagem em tempos de pandemia, durante o cuidado prestado à clientela.

\section{Espiritualidade: importante dimensão do cuidado}

A Enfermagem é vista como a linha de frente nessa pandemia e o medo e a incerteza que permeiam essa doença são fatores capazes de gerar estresse emocional em muitos profissionais de enfermagem ${ }^{(7)}$. O medo dificulta o enfrentamento das situações que se apresentam novas no cotidiano, paralisando algumas vezes as atitudes e ações da vida diária, o que pode interferir no cuidado direto.

Nessa perspectiva, a espiritualidade é uma ferramenta facilitadora do cuidar, sobretudo em situações em que a doença se mostra sem cura e/ou em situações de desespero dos pacientes e familiares ${ }^{(8)}$.

A espiritualidade tem se revelado um dos principais recursos dos profissionais e da sociedade para compreender os sofrimentos e fortalecer a humanidade para novos desafios como à COVID-19. O isolamento do paciente portador ou suspeito dessa enfermidade, até mesmo no momento de dor e sofrimento, reforça a necessidade da abordagem da espiritualidade como uma dimensão importante a ser considerada nesse cuidado.

Desse modo, a espiritualidade, entendida na sua essência como uma dimensão fundamental do ser humano, encontra, nos cenários da saúde, cada vez mais um lugar inquestionável, em especial no âmbito de uma pandemia de transmissão rápida, com angústia respiratória e que requer a adoção de precauções bastante acentuadas ${ }^{(6,9)}$.

Para muitos pacientes, a espiritualidade e religiosidade são vistas como uma forma de enfrentamento da doença com a finalidade de minimizar o sofrimento e obter maior esperança, impactando na sua qualidade de vida, destacando assim a importância da ferramenta espiritual na assistência ${ }^{(10)}$. 
A espiritualidade é um importante pilar no processo de ressignificação do processo de morte/morrer e luto de um modo geral, mas no caso da COVID-19 se reveste de considerável importância em função do medo generalizado, do elevado número de mortes diárias e das constantes teorias "ingênuas" construídas, como a do fim do mundo, de castigo divino e de sua previsão em livros sagrados ${ }^{(6,10)}$.

\section{A Unidade de Terapia Intensiva como cenário do cuidado es- piritualizado}

Devido às complicações advindas da COVID-19, muitos pacientes acabam por serem transferidos para as Unidades de Terapia Intensiva (UTI), e alguns necessitam de sedação e intubação. E como utilizar-se das dimensões espirituais nesse momento? Quais benefícios trariam para o cuidado desse paciente e para o profissional cuidador? No contexto do cuidado, a espiritualidade é reconhecida como componente que humaniza o cuidado e pode auxiliar nas relações cuidador e ser cuidado(6)

Em muitos casos, os profissionais da saúde que atuam nas UTI, convivem diariamente com a morte, porém a situação atual da pandemia por coronavírus é capaz de reacender pensamentos e questões psicológicas frente à morte e ao morrer que até então estavam esquecidas ou adormecidas no interior desses profissionais, culminando no surgimento de grande estresse psicológico. Assim, as experiências espirituais são essenciais, tanto em quem cuida, quanto no outro que está a sendo cuidado, sobretudo nesse setor, visto que se trata de unidade crítica, onde a vida muitas vezes está severamente ameaçada ${ }^{(11)}$.

Quanto maior a espiritualidade dos profissionais da saúde que atuam em UTI, maior é o reconhecimento da influência positiva para a recuperação da pessoa assistida nesse setor ${ }^{12}$. A valorização da dimensão espiritual do paciente assistido neste ambiente pode colaborar com a sua recuperação, melhor entendimento e aceitação da sua condição atual, contribuindo para um melhor equilibrio e qualidade de vida, preservando sua dignidade durante o período de internação(12).

\section{Como inserir a espiritualidade e religiosidade no cuidado?}

A introdução da espiritualidade no cuidado a esses pacientes traz consigo dois aspectos importantes a serem considerados: a falta de treinamento dos profissionais de enfermagem, bem como a variabilidade de crença entre quem cuida e quem está sendo cuidado, sendo assim, se faz necessário a separação entre a espiritualidade e religiosidade nesse momento.

A distinção entre espiritualidade e religiosidade no momento do tratamento pode auxiliar na resolução desse pro- blema, ou seja, concentra-se na espiritualidade de maneira geral ao invés de concentrar-se em um sistema de crenças que nem sempre é de conhecimento do profissional(8,12-13)

Discussão, reflexão e ações que possibilitem que o enfermeiro introduza na sua prática diária as questões inerentes à espiritualidade, é uma necessidade. A inserção da entrevista espiritual na sistematização da assistência de Enfermagem a fim de que logo no início do estabelecimento do contato/ vínculo do ser cuidado com o cuidador fosse possível levantar suas crenças e valores, visando suprir as necessidades inerentes a este campo no acompanhamento do indivíduo no seu processo de saúde e doença ${ }^{(14)}$.

A espiritualidade é algo intrínseco a natureza do homem e com poder de cura, entretanto ao longo de décadas os indivíduos foram se afastando desses pilares, objetivando a técnica e o cientificismo, abandonando a herança religiosa e a dimensão espiritual do cuidado(14)

A Teoria de Enfermagem das Necessidades Humanas Básicas de Wanda de Aguiar Horta coloca a espiritualidade como uma dimensão a ser considerada, bem como o Diagnóstico de Enfermagem "angústia espiritual" proposto pela North American Nursing Diagnosis Association (NANDA)(15).

\section{Limitação do estudo}

Este estudo apresenta como limitação, o número reduzido de artigos que abordam a temática COVID-19 e a espiritualidade e religiosidade como ferramentas de suporte para o cuidado de Enfermagem.

\section{Contribuições do estudo para a prática}

Espera-se que este estudo traga contribuições para o ensino, pesquisa e prática de Enfermagem frente aos pacientes com COVID-19, uma vez que no estudo traz subsídios para a utilização da espiritualidade como ferramenta de suporte no cuidar de modo a fortalecer os profissionais de Enfermagem e pacientes no enfrentamento desta pandemia. Além disso, este estudo estimula o desenvolvimento de outras pesquisas sobre a temática, sobretudo no que tange o cuidado de Enfermagem frente a pacientes portadores da COVID-19.

\section{CONSIDERAÇÕES FINAIS}

Diante do atual cenário de pandemia, a espiritualidade aponta para o caminho da esperança, da resiliência, da reflexão diante de vários aspectos, individuais e coletivos que precisam ser trabalhados para que os recursos internos possam ser acessadospara passar pela situação atual

A dimensãoda espiritualidade nocuidar reforça o conceito de integralidade em saúde, uma vez que envolvem, no cuidado, outras dimensões que não somente a saúde e doença, transforma o cuidado em algo que excede a questão técnica. 
Ao mesmo tempo, a presença da espiritualidade em saúde reforça e estimula a integralidade no cuidar, colaborando com maiores níveis de humanização em saúde.

Em virtude do papel que a espiritualidade pode ter na doença, vida e qualidade de vida do paciente, é importante que profissionais de enfermagem estejam habilitados a explorar essa faceta dos seus clientes como também em si mesmo.

Todavia, a maior parte dos profissionais de enfermagem sentem-se despreparados para lidar com situação que envolve a espiritualidade e saúde, evidenciando que aimplementação de dimensões espirituais no cuidar não é relativamente fácil, sendo necessário que este seja qualificado de modo a se sentir seguro para prestar cuidado humanizado e integral.

\section{CONTRIBUIÇÕES DOS AUTORES}

Diogo foi responsável pela concepção e desenho do estudo, delimitando os passos, objetivos de pesquisa e metodologia a ser empregada. Márcia foi responsável por toda parte de coleta, e separação dos dados em categorias para facilitar a análise e interpretação de dados. Marcos foi responsável pela redação do manuscrito e revisão crítica. Fabiana foi responsável pela revisão crítica, formatação do manuscrito nas normas e aprovação da versão final

\section{REFERÊNCIAS}

1.Ministério da Saúde (BR). Protocolo de Manejo Clínico para o Novo Coronavirus - 2019-nCOV. Ministério da Saúde [internet] 2020 [cited 2020 Maio 10]; Available from: https://portalarquivos2.saude.gov.br/images/ pdf/2020/fevereiro/11/protocolo-manejo-coronavirus.pdf

2.Ministério da Saúde (BR). Boletim Epidemiológico 17 - Doença pelo Coronavirus 2019. Ministério da Saúde [Internet]. 2020 [cited 2020 Maio 23] 1(1):1-6. Available from: https://central3.to.gov.br/arquivo/502380/.

3.Crispin D, da Silva MJP, Cedotti W. Milena C, Gomes AS. Comunicação Dificil e COVID-19: Recomendações práticas para comunicação e acolhimento em diferentes cenários da pandemia. Associação Médica de Minas Gerais [internet]. 2020 [cited 2020 Maio 20]; 1(1):17-28. Available from: https://ammg. org.br/wp-content/uploads/comunica\%C3\%A7\%C3\%A3o-COVID-19.pdf. pdf.

4. Mary RGE, Renate BM, Trebien HAC, Menegatti CL. Coping Religioso/Espiritual na Antessala de UTI: Reflexões sobre a Integração da Espiritualidade nos Cuidados em Saúde. Revista Interações [Internet]. 2017 [cited 2020 Maio 20]:12(22):303. Available from: http://periodicos.pucminas.br/index. php/interacoes/article/view/P.1983-2478.2017v12n22p303/12912

5.Barbosa DJ, Gomes AMT, Soares GO. Paes LS. Religiosidade e Espiritualidade como ferramenta de apoio para o tratamento de usuários de drogas psicoativas. Revista Próuniver-SUS. [internet] 2018. [cited 2020 Maio 06] 09(2):17-23. Available from: http://editora.universidadedevassouras.edu.br/ index.php/RPU/article/view/1432

6.Tavares CO. Dimensões do cuidado na perspectiva da espiritualidade durante a pandemia pelo novo coronavirus (COVID-19). Journal Health NPEPS. [internet] 2020 [cited 2020 Maio 1]; 5(1):1-4. Available from: https:// pesquisa.bvsalud.org/portal/resource/pt/biblio-1095168

7.Barbosa DJ, Gomes MP, Souza FBA, Gomes AMT. Fatores de estresse nos profissionais de enfermagem no combate à pandemia da COVID-19: Sintese de Evidências. Comunicação em Ciências da Saúde. [internet] 2020. [cited 2020 Maio 02]; 31(1):31-47. Available from: http://www.escs.edu.br/revistaccs/index.php/comunicacaoemcienciasdasaude/article/view/651

8.Chirelli M. Mishima S. A formação do enfermeiro crítico-reflexivo no curso de enfermagem da Faculdade de Medicina de Marilia - FAMEMA. Revista Latino-Americana de Enfermagem. [internet] 2003 [cited 2020 Maio 05]

1l(5):574-584. Available from: https://www.scielo.br/scielo.php?pid=S0104 -11692003000500003\&script=sci_arttextEtlng=pt

9.Baczewska B, Block B, Kropornicka B, Niedzielski A, Malm M, Zwolak A et al. Hope in Hospitalized Patients with Terminal Cancer. International Journal of Environmental Research and Public Health. [internet] 2019 [cited 2020 Maio 05]; 16(20):3867. Available from: https://www.ncbi.nlm.nih.gov/ pmc/articles/PMC6844130/

10.Penha RM, Silva, Maria JP. Significado de espiritualidade para a enfermagem em cuidados intensivos. Texto \& Contexto - Enfermagem. [internet] 2012 [cited 2020 Maio 10]; 21(2):260-268. Available from: https://www.scielo. br/scielo.php?pid=S0104-07072012000200002Escript=sci_abstractEtl$\mathrm{ng}=\mathrm{pt}$

11.Vale CCSO, Libero ACA. A espiritualidade que habita o CTI. Periódicos Eletrônicos em Psicologia. [internet] 2017 [cited 2020 Maio 10]; 11(21):321328. Available from: http://pepsic.bvsalud.org/scielo.php?script=sci_arttextEpid=S1679-44272017000200003.

12.De La Longuiniere A, Donha Yarid S, Sampaio Silva E. Influência da religiosidade/espiritualidade do profissional de saúde no cuidado ao paciente crítico. Revista Cuidarte [Internet]. 2018 [cited 2020 Maio 21]; 9(1):1961. Available from: https://revistacuidarte.udes.edu.co/index.php/cuidarte/ article/view/413.

13.Guilherme L. A religiosidade/espiritualidade na prática do cuidado entre profissionais da saúde. Interações [Internet]. 2016 [cited 2020 Maio 21]; 11(20):129. Available from: http://periodicos.pucminas.br/index.php/interacoes/article/view/P.1983-2478.2016vlln20p129.

14.Raddatz JS, Motta RF, Alminhana LO. Religiosidade/Espiritualidade na prática clinica: círculo vicioso entre demanda e ausência de treinamento. Psico-USF [Internet]. 2017 [cited 2020 Maio 21]; 24(4):699-709. Available from http://www.scielo.br/scielo.php?script=sci_arttextEpid=S1413-82712019000400699\&lng=en\&nrm=iso

15.Xavier ECL, Correa AJS, Carvalho MMC, Lima FR, Santana ME. Diagnósticos de enfermagem em cuidados paliativos oncológicos segundo diagrama de abordagem multidimensional. Enferm Foco [Internet]. 2019 [cited 2020 Maio 21]; 10(3):157-157. Available from: http://revista.cofen.gov.br/index. php/enfermagem/article/view/2109/569. 